\title{
Angiotensin-(1-7)-Mediated Signaling in Cardiomyocytes
}

\author{
Enéas R. M. Gomes, ${ }^{1}$ Robson A. S. Santos, ${ }^{1,2}$ and Silvia Guatimosim ${ }^{1,2}$ \\ ${ }^{1}$ Department of Physiology and Biophysics, Institute of Biological Sciences, Federal University of Minas Gerais, \\ 31270-901 Belo Horizonte, MG, Brazil \\ ${ }^{2}$ National Institute of Science and Technology in Nanobiopharmaceutics, Institute of Biological Sciences, \\ Federal University of Minas Gerais, 31270-901 Belo Horizonte, MG, Brazil
}

Correspondence should be addressed to Silvia Guatimosim, guatimosim@icb.ufmg.br

Received 1 September 2011; Revised 22 November 2011; Accepted 24 November 2011

Academic Editor: Anderson J. Ferreira

Copyright (C) 2012 Enéas R. M. Gomes et al. This is an open access article distributed under the Creative Commons Attribution License, which permits unrestricted use, distribution, and reproduction in any medium, provided the original work is properly cited.

\begin{abstract}
The Renin-Angiotensin System (RAS) acts at multiple targets and has its synthesis machinery present in different tissues, including the heart. Actually, it is well known that besides Ang II, the RAS has other active peptides. Of particular interest is the heptapeptide Ang-(1-7) that has been shown to exert cardioprotective effects. In this way, great compilations about Ang-(1-7) actions in the heart have been presented in the literature. However, much less information is available concerning the Ang-(1-7) actions directly in cardiomyocytes. In this paper, we show the actual knowledge about Ang-(1-7)-mediated signaling in cardiac cells more specifically we provide a brief overview of ACE2/Ang-(1-7)/Mas axis; and highlight the discoveries made in cardiomyocyte physiology through the use of genetic approaches. Finally, we discuss the protective signaling induced by Ang-(1-7) in cardiomyocytes and point molecular determinants of these effects.
\end{abstract}

\section{Introduction}

Arterial hypertension is an important cardiovascular risk factor and contributes to the development of cardiovascular event. Despite the substantial advances in antihypertensive drug therapy, the number of patients with uncontrolled hypertension remains high around the world [1].

The renin-angiotensin system (RAS) is an important classical player that directly contributes to the development and maintenance of essential hypertension [2]. This system is classically known as a hormonal system, involved in salt and water regulation and blood pressure control. Angiotensin (Ang) II, one of the main components of the RAS, exerts its biological effects by binding with high affinity to two distinct subtypes of receptor, the angiotensin II type 1 receptor $\left(\mathrm{AT}_{1} \mathrm{R}\right)$ and the angiotensin II type 2 receptor $\left(\mathrm{AT}_{2} \mathrm{R}\right)[3,4]$. Under physiological and pathological states, it is recognized that $\mathrm{AT}_{1} \mathrm{R}$ plays a critical role in Ang II-mediated actions in the cardiovascular system $[3,4]$. On the other hand, a large body of evidence suggests that $\mathrm{AT}_{2} \mathrm{R}$ antagonizes the effects of $\mathrm{AT}_{1} \mathrm{R}$ preventing, between other effects, its hypertrophic and angiogenic effects $[5,6]$.

Although Ang II is the major effector of this system, several other peptides are now recognized as being biologically important. Of particular importance is the heptapeptide Ang-(1-7) that decades ago emerged as a new metabolite of the RAS. Ang-(1-7) was initially detected as an Ang I metabolite in canine brain homogenates [7]. This discovery led to the later demonstration of its action in releasing vasopressin from hypophyseal-hypothalamic explants [8] and in counteracting the pressor and baroreflex effects of Ang II [9-11]. Later on, Ang-(1-7) was finally recognized as a putative biologically active component of the RAS [12-14]. Since then, the physiological actions of Ang-(1-7) have been extensively investigated. The heart is an important target for Ang-(1-7), which exerts direct effects on cardiomyocytes. The following sections focus on the cellular mechanism and signaling pathways involved in Ang-(1-7) actions in the cardiac cell, with particular emphasis on recent discoveries made through the use of genetic approaches. 


\section{The Angiotensin-Converting Enzyme2/Angiotensin-(1-7)/Mas Axis}

Ang-(1-7) can be formed directly from Ang I or Ang II and indirectly from Ang I having as an intermediate step the formation of Ang-(1-9) [14, 15]. Angiotensin-converting enzyme (ACE) 2, as well as prolylcarboxypeptidase (PCP) and prolylendopeptidase (PEP), can generate Ang-(1-7) directly from Ang II. Apparently, the principal enzyme and pathway involved in the Ang-(1-7) generation is ACE2 through the hydrolysis of Ang II [16, 17]. However, Campbell et al. [18] have suggested that PEP is the main enzyme responsible for generating Ang-(1-7) from Ang II in human coronary vessels. In addition, it appears that the generation of Ang-(1-7) involving an intermediate step, including the hydrolysis of Ang I to Ang-(1-9), is less important [19]. Ang(1-7) is also produced directly from Ang I by neutral endopeptidase (NEP) and PEP through hydrolysis of the Pro7Phe8 bond $[19,20]$. A schematic representation of the enzymatic pathways involved in the generation of Ang-(1-7) is presented in Figure 1.

In cardiomyocytes the expression of ACE2, the main enzyme involved in Ang-(1-7) formation, was already demonstrated [21, 22], as well as NEP [23]. In addition, ACE mRNA has been consistently demonstrated in cardiac cells $[24,25]$. However, the ACE mRNA levels were not supported by protein measurements in human hearts. No immunoreactivity for ACE was found in ventricular myocytes from human control hearts, with ACE detected only in cardiomyocytes from hearts after myocardial infarction [26]. Nevertheless, ACE has been demonstrated in cardiomyocytes from rats and mice by enzyme-kinetic and immunohistochemical methods [27]. The presence in the cardiomyocyte of these specific enzymes indicates that Ang(1-7) and some of RAS components can be locally synthesized in the heart. It remains to be determined which components are produced locally and in which conditions this production is activated.

The identification of ACE2 as an important Ang-(1-7)forming enzyme $[28,29]$, and of Mas as a G protein-coupled receptor for Ang-(1-7) [30], contributed to establish Ang-(17) as a biologically active component of the RAS. In 2005, Ferreira and Santos advanced the hypothesis that ACE2, Ang(1-7), and Mas could be considered as components of a novel axis of the RAS, the so-called ACE2/Ang-(1-7)/Mas axis [31]. For the heart, this concept is now quite well accepted in the literature $[13,32-35]$. But, at present, only limited information is available regarding the direct effects of ACE2/Ang-(1-7)/Mas axis activation for cardiomyocyte function during physiological as well as in pathological conditions. In addition to ACE2, the presence of Mas has been demonstrated in cardiomyocytes from different species $[21,22,36,37]$, including humans [38].

A variety of vasoactive peptides and hormones can regulate ACE2 mRNA levels in cardiomyocytes. Modulation of ACE2 mRNA levels by aldosterone has been demonstrated in neonatal cardiomyocytes treated with this mineralocorticoid [22]. Accordingly, aldosterone decreased ACE2 mRNA levels in these cells, an effect apparently mediated by the mineralocorticoid receptor. In the same study, ACE2 mRNA modulation was not affected by Ang II treatment, suggesting that ACE2 mRNA expression is under differential modulation by endocrine molecules in cardiomyocytes. Considering the Ang II actions on ACE2 mRNA levels, opposing results were obtained by Gallagher et al. [21], who found a decrease in ACE2 activity and downregulation of its mRNA by Ang II. Importantly, this effect was mediated by $\mathrm{AT}_{1} \mathrm{R}$ and blocked by inhibitors of mitogen-activated protein kinase kinase 1 (MAPKK1). Considering that differences in experimental conditions can explain the contrasting results regarding Ang II modulation of ACE2, further investigation will be necessary to elucidate the specific mechanism involved in ACE2 downregulation in cardiomyocytes. Endothelin-1 (ET-1) also significantly reduced myocyte ACE2 mRNA via MAPKK1 activation [21]. Apparently Ang-(1-7) has no direct effect on ACE2 mRNA regulation, although this peptide, through Mas receptor, blocked the Ang II and ET-1 mediated downregulation of ACE2 expression [21]. Collectively, these results indicate that ACE2 expression in cardiomyocytes is tightly regulated by important modulators of cardiovascular system, highlighting its importance in cardiac disease establishment and progression. Since ACE2 converts Ang II to Ang-(1-7), it is plausible that ACE2 downregulation by Ang II serves as a mechanism to favor Ang II-mediated responses, by preventing its degradation to Ang-(1-7). Thus, conditions favoring excess Ang II generation and reduced Ang II breakdown would likely lead to more deleterious effects on the heart. Figure 2 summarizes the information regarding the modulation of ACE2 expression in cardiomyocytes.

\section{Protective Signaling Induced by ACE2/Ang-(1-7)/Mas Axis in Cardiomyocytes}

In the past two decades, since the detection of the Ang-(1-7) as a product of the metabolism of Ang I, the physiological actions of Ang-(1-7) have been extensively investigated, and Ang-(1-7) was finally recognized as a putative biologically active component of the RAS [12-14]. However, besides this great advance in the understanding of Ang-(1-7) actions, especially in the heart, only a few reports have explored the Ang-(1-7) actions directly in cardiomyocytes. This section will focus on signaling pathways and molecular determinants of Ang-(1-7) signaling in cardiomyocytes. This will be accomplished by highlighting the following effects: (1) antihypertrophic, (2) anti-inflammatory, and (3) antioxidative. In addition, we summarize current knowledge regarding Ang-(1-7) modulation of $\mathrm{Ca}^{2+}$ handling in cardiomyocytes. Initial studies were performed by Tallant et al. [39], who confirmed the presence of the Mas receptor in neonatal cardiomyocytes and showed a direct effect of Ang-(1-7) in these cells, by preventing cell growth, through inhibition of the MAPK ERK1/2 activity. Later on, it was demonstrated the presence of Mas receptor in adult ventricular myocytes [40]. Continuing the exploration of Ang-(1-7) actions and pathways in cardiomyocytes, some information coming from 


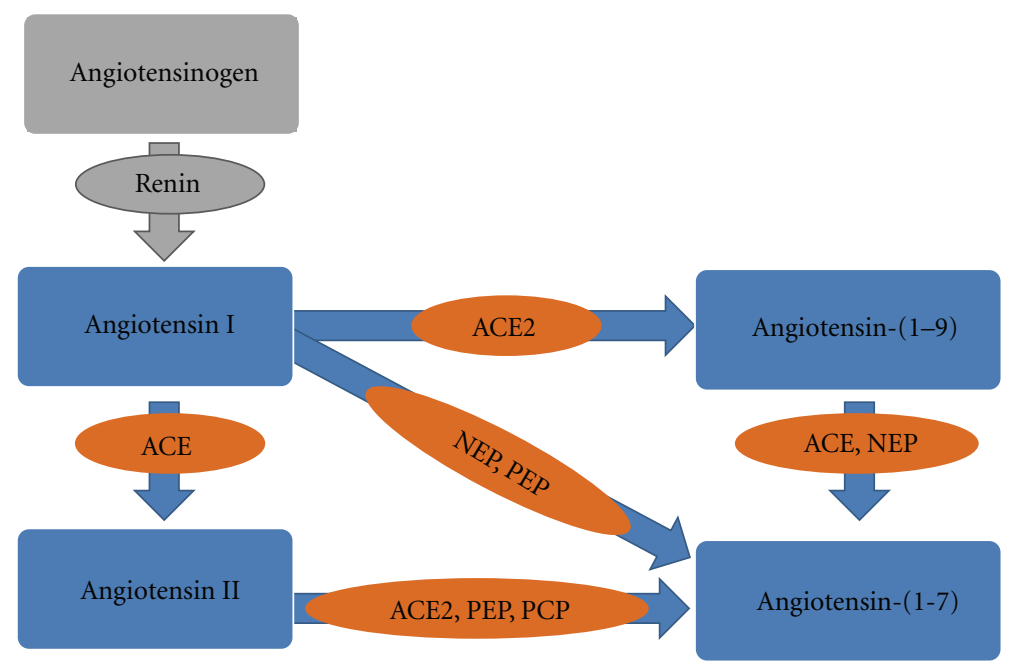

FIGURE 1: Schematic representation of the enzymatic pathways involved in the generation of Ang-(1-7). Ang-(1-7) can be formed by at least three different pathways: directly from Ang I by NEP and PEP, by hydrolysis of Ang II by ACE2, PEP, and PCP, and finally by hydrolysis of Ang-(1-9) by ACE and NEP. ACE, ACE2, and NEP are found in cardiomyocytes.

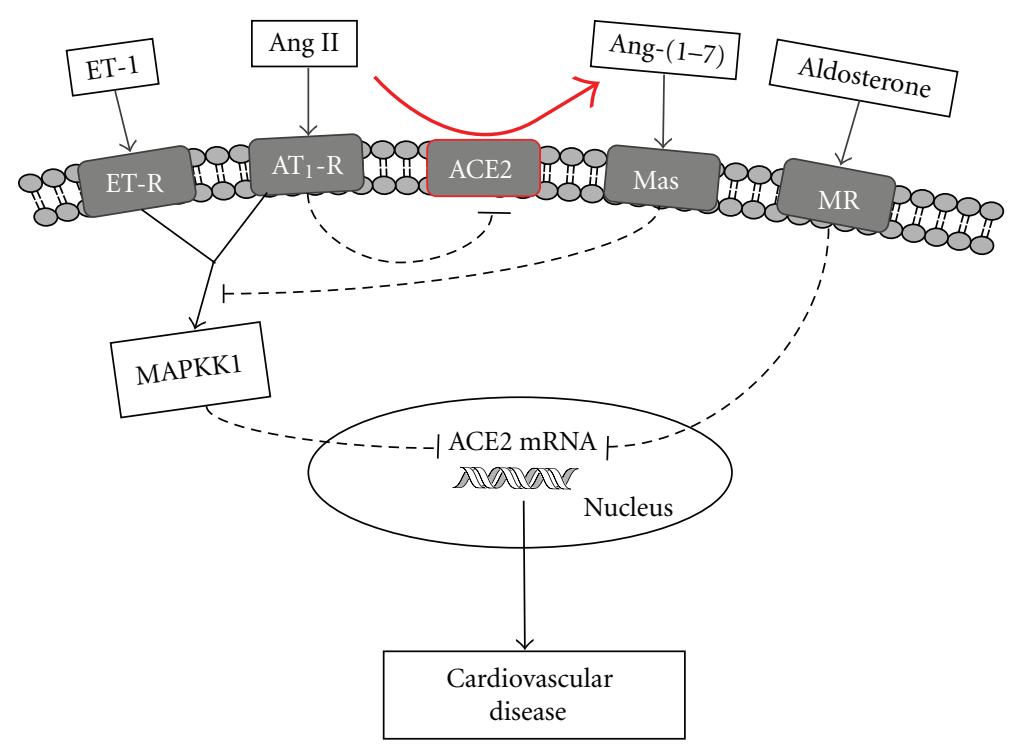

FIGURE 2: Schematic illustration of ACE2 modulation in cardiomyocytes by different molecules. Ang II, and ET-1 through MAPKK1 activation, and aldosterone lead to ACE2 mRNA downregulation. To date no reported effects of Ang-(1-7) on ACE2 levels have been demonstrated, although this peptide is capable of antagonizing Ang II and ET-1 effects on ACE2 mRNA. Conditions that favor ACE2 downregulation would likely lead to more deleterious effects during cardiovascular disease development. $\rightarrow=$ activation; $--\dashv=$ inhibition; ET-1 = endothelin; Ang II = angiotensin II; Ang-(1-7) = angiotensin-(1-7); ET-R = endothelin receptor; $\mathrm{AT}_{1}-\mathrm{R}=\mathrm{AT}_{1}$ receptor; Mas = Mas receptor; $\mathrm{MR}=$ mineralocorticoid receptor; $\mathrm{MAPKK} 1=$ mitogen-activated protein kinase kinase 1 .

different types of cell supported the next steps in the understanding of the signaling molecules involved in the Ang-(17) effects. Sampaio et al. [41] showed that in endothelial cells Ang-(1-7) was able to generate nitric oxide (NO). In the same way, Dias-Peixoto et al. [36] demonstrated that Ang-(1-7) was able to activate the phosphatidylinositol 3kinase (PI3-K)-protein kinase B (Akt)-pathway, resulting in nitric oxide synthase (NOS) 3 activation and NO generation in adult ventricular cardiomyocytes. It should be noted that cardiomyocytes express distinct subtypes of PI3-K, and some of them are activated by Ang II $[42,43]$. Therefore, is of particular importance to investigate which specific pools of PI3-K are regulated by Ang II and Ang-(1-7). In addition, Dias-Peixoto et al. [36] have shown that expression levels 


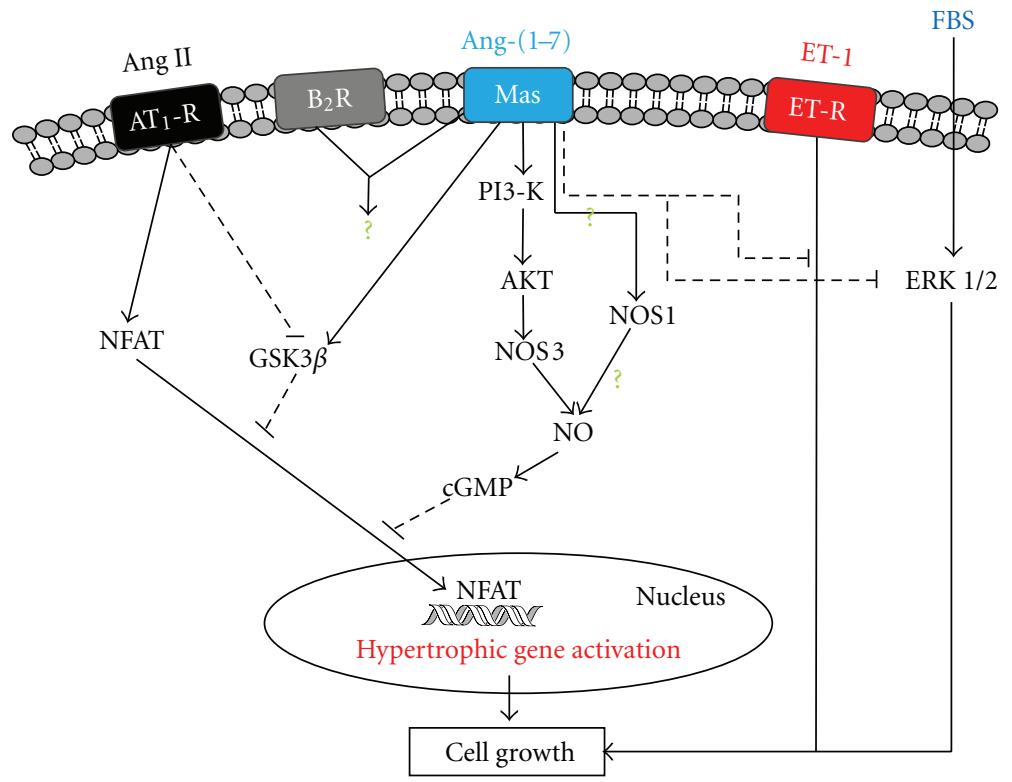

FIgURE 3: Ang-(1-7) signaling in cardiomyocytes. Ang-(1-7) activates the PI3-K/AKT/NOS3 pathway leading to NO generation and cGMP production. Activation of this pathway culminates with inhibition of Ang II-induced NFAT translocation. Preliminary evidence suggests that NOS1 expression may be modulated by Ang-(1-7). Ang-(1-7) also inhibits ET-1 and FBS activation of cell growth. The consequences of Ang-(1-7)/Mas and $B_{2} \mathrm{R}$ cross-talk for cardiomyocyte function are still unknown (?). $\rightarrow=$ activation; $--\dashv=$ inhibition; ET-1 = endothelin; Ang II = angiotensin II; Ang-(1-7) = angiotensin-(1-7); ET-R = endothelin receptor; $\mathrm{AT}_{1}-\mathrm{R}=\mathrm{AT}_{1}$ receptor; Mas = Mas receptor; $\mathrm{B}_{2} \mathrm{R}=$ bradykinin receptor type 2 ; FBS = fetal bovine serum; NFAT = nuclear factor of activated T cells; GSK3 $\beta=$ glycogen synthase kinase $3 \beta$; $\mathrm{PI} 3-\mathrm{K}=$ phosphatidylinositol 3-kinase; $\mathrm{AKT}=$ protein kinase $\mathrm{B}$; NOS3 = nitric oxide synthase 3 ; NOS1 = nitric oxide synthase 1 ; NO = nitric oxide; cGMP = cyclic guanosine monophosphate; ERK 1/2 = extracellular signal regulated kinase 1/2. The question marks denote areas in which the current state of knowledge is still preliminary.

of proteins involved in the NOS3 macromolecular complex, such as caveolin-3, heat shock protein (HSP)-90, and protein kinase B (AKT), were altered in ventricular myocytes from $\mathrm{Mas}^{-/-}$(Mas knockout) mice, indicating an important relationship between NOS3 activity and Ang-(1-7)/Mas axis. Initial investigation into the cellular mechanisms underlying protective effects of Ang-(1-7) against Ang II signaling was recently performed by our laboratory. Gomes et al. [37] have demonstrated, in cardiomyocytes, that Ang-(1-7) prevention of Ang II-induced pathological remodeling is mediated by NO/cGMP (cyclic guanosine monophosphate) pathway. This result identifies a role of $\mathrm{NO}$ as mediator of Ang-(1-7) beneficial effects and extends the concept that cGMP is another key molecule in this signaling pathway. In addition, this study showed that transgenic rats presenting increased Ang-(1-7) plasmatic levels have higher levels of NOS1 in ventricular cardiomyocytes, showing that besides NOS3, NOS1 shall be involved in NO generation elicited by Ang-(17). Ang-(1-7) also modulated the activity of the transcription factor NFAT (nuclear factor of activated T cells), preventing its translocation to the nucleus, and the activation of hypertrophic gene program by Ang II [37]. Stimulated by calcium signals, NFAT is translocated to the nucleus where it can regulate hypertrophic genes. In cardiomyocytes, NFAT nuclear localization is tightly controlled at multiple levels [44-46]. Glycogen synthase kinase $3 \beta$ (GSK3 $\beta$ ), in particular, is considered a potent inhibitor of this pathway downstream of calcineurin. In the nucleus, GSK3 $\beta$ phosphorylates NFAT, thereby promoting its nuclear export [44]. Moreover, GSK3 $\beta$ has been shown to regulate hypertrophy development by restraining gene expression [47]. Gomes et al. [37] have shown that Ang-(1-7) modulates the activity of GSK3- $\beta$, by preventing its inactivation by Ang II. The modulation of these two proteins, NFAT and GSK3- $\beta$, supports the anti-hypertrophic effect of Ang-(1-7) observed in the heart [37] and in cardiomyocytes [37, 39]. Corroborating these findings Flores-Muñoz et al. [48] reported that Ang-(1-7) was able to block the increase in cell size induced by Ang II in H9c2 cardiomyocytes. These effects were mediated by Mas receptor, since Mas antagonist A779 efficiently blocked the antihypertrophic effects of Ang-(1-7). Importantly, these authors have also shown that Ang-(1-7) anti-hypertrophic activity was inhibited in the presence of the bradykinin type 2 receptor $\left(\mathrm{B}_{2} \mathrm{R}\right)$ antagonist, HOE140, suggesting a crosstalk between Mas and $B_{2} R$ in response to Ang-(1-7). Figure 3 shows recent data about Ang-(1-7) signaling and cross-talk in cardiomyocytes.

Recently, Qi et al. have provided evidence for an antiinflammatory role of angiotensin-(1-7) at the cardiomyocyte level [49]. By using neonatal cardiomyocyte culture, the authors demonstrated that protective effects of Ang-(1-7) against hypoxia-induced cell death were mediated, at least in part, through modulation of cytokine production. This beneficial effect was associated with decreased expression 
of inflammatory cytokines such as tumor necrosis factor- $\alpha$ (TNF- $\alpha$ ) and interleukin-6 (IL-6) and increased gene expression of ACE2, bradykinin type 2 receptor, and interleukin10 (IL-10). Taken together, these data show that Ang-(1-7) regulates cytokine responses, which could contribute to its cardioprotective effects.

Considering the critical role of $\mathrm{Ca}^{2+}$ ions for cardiomyocyte contraction [50], some studies have addressed whether Ang-(1-7) modulates $\mathrm{Ca}^{2+}$ handling in ventricular cardiomyocytes. Recent work by our group has shown that acute Ang(1-7) treatment does not significantly alter $\mathrm{Ca}^{2+}$ transient amplitude or kinetics of decay [36]. We extended these findings to in vivo conditions and showed that cardiomyocytes from transgenic (TG) rats with chronic elevated plasmatic Ang-(1-7) do not show alteration in cytosolic $\mathrm{Ca}^{2+}$ transient parameters [37]. Interestingly, cardiomyocytes from mice with genetic ablation of Ang-(1-7) Mas receptor $\left(\mathrm{Mas}^{-/-}\right)$ presented a $\mathrm{Ca}^{2+}$ signaling dysfunction represented by a smaller peak $\mathrm{Ca}^{2+}$ transient and slower $\mathrm{Ca}^{2+}$ uptake. This $\mathrm{Ca}^{2+}$ signaling dysfunction was accompanied by decreased protein levels of the sarcoplasmic/endoplasmic reticulum $\mathrm{Ca}^{2+}$ ATPase 2 (SERCA2) [36]. SERCA2 is responsible for $\mathrm{Ca}^{2+}$ reuptake by the sarcoplasmic reticulum (SR), thereby setting SR $\mathrm{Ca}^{2+}$ load, which is an important determinant of $\mathrm{Ca}^{2+}$ release in cardiomyocytes [50]. The reduction in the $\mathrm{Ca}^{2+}$ transient is consistent with the depression of contractility that was previously observed in $\mathrm{Mas}^{-/-}$hearts [40]. This finding was particularly important since it suggested that the Ang-(1-7)/Mas axis is critical for long-term maintenance of normal $\mathrm{Ca}^{2+}$ handling in the cardiac cell. However, there was still the possibility that the alterations in $\mathrm{Ca}^{2+}$ handling found in $\mathrm{Mas}^{-/-}$cardiomyocytes were secondary to the cardiac dysfunction observed in these hearts.

Adding further complexity to the understanding of Ang(1-7)/Mas modulation of cardiomyocyte $\mathrm{Ca}^{2+}$ signaling, it was also shown that cardiomyocytes from TG rats with cardiac specific overexpression of Ang-(1-7) presented higher $\mathrm{Ca}^{2+}$ transient amplitude, faster $\mathrm{Ca}^{2+}$ uptake, and increased levels of SERCA2 [51], suggesting that chronic local increase of Ang-(1-7) in the heart was associated to enhanced $\mathrm{Ca}^{2+}$ handling. Are these changes in $\mathrm{Ca}^{2+}$ handling a direct consequence of local Ang-(1-7) increase in the heart? These findings contrasted with the lack of effect on $\mathrm{Ca}^{2+}$ signaling found in cardiomyocytes from TG rats with chronic elevated plasmatic Ang-(1-7) levels. As it stands, the relationship between Ang-(1-7) and $\mathrm{Ca}^{2+}$ signaling is more complex than one may have anticipated. It is also plausible, that Ang-(1-7) effects on $\mathrm{Ca}^{2+}$ handling observed in an in vivo model of chronic Ang-(1-7) overexpression in the heart are consequences of long-term changes in expression levels of $\mathrm{Ca}^{2+}$ handling proteins. Future studies are needed to demonstrate whether Ang-(1-7) prevents $\mathrm{Ca}^{2+}$ signaling dysfunction in ventricular myocytes from animal models of heart failure.

NO has been attributed as a key mediator of Ang-(1-7) effects on different cell types, including cardiomyocytes, and it is known to interact with proteins involved in $\mathrm{Ca}^{2+}$ handling and regulate cardiac contractility. The question remains whether long-term Ang-(1-7) effects on $\mathrm{Ca}^{2+}$ handling are mediated by NO or other signaling molecules in cardiomyocytes. In this way, the regulation of some key proteins involved in cardiomyocyte $\mathrm{Ca}^{2+}$ handling, such as ryanodine receptor (RyR), phospholamban (PLN), $\mathrm{Na}^{+} / \mathrm{Ca}^{2+}$ exchanger (NCX), and troponins by Ang-(1-7) must be investigated in order to provide a deeper understanding of Ang-(1-7) actions on $\mathrm{Ca}^{2+}$ signaling. A summary of current knowledge regarding Ang-(1-7)/Mas modulation of cardiomyocyte $\mathrm{Ca}^{2+}$ signaling is shown in Figure 4.

Evidence for a direct role of ACE2/Ang-(1-7)/Mas axis against oxidative stress in cardiomyocytes was also obtained [52]. Experiments on adult ventricular myocytes demonstrated that Ang II-mediated superoxide generation and extracellular signal-regulated kinase $1 / 2$ (ERK 1/2) activation were inhibited by recombinant ACE2 (rhACE2). Importantly, these effects were mediated by Ang-(1-7), since preincubation with the Mas receptor peptide antagonist, D-Ala7-Ang-(1-7), largely prevented rhACE2 suppression of Ang II-induced responses in cardiomyocytes. These in vitro findings correlated with in vivo data showing that treatment with rhACE2 prevented Ang II-induced hypertrophy and myocardial fibrosis. Thus, these findings give further support to the fact that enhanced Ang-(1-7) signaling at the cardiomyocyte level prevents Ang II pathological effects and highlight Ang-(1-7) anti-oxidative actions on cardiomyocytes.

Contrasting to the view of a protective role of ACE2/Ang(1-7)/Mas axis some reports have shown hypertrophic effects of ACE2 and Mas overexpression in cardiomyocytes. Masson et al. [53] reported that adenoviral-mediated gene transfer of ACE2 in rabbit cardiomyocytes leads to cellular hypertrophy. In the same study, in vivo ACE2 overexpression in the myocardium of stroke-prone spontaneously hypertensive rats resulted in profound cardiac dysfunction. The authors have argued that the detrimental effects of ACE2 overexpression were possibly due to higher amounts of protein expressed using this approach. Using similar overexpression strategy, neonatal rat cardiomyocytes were infected with adenovirus encoding the human Mas receptor. Intriguingly, overexpression of Mas induced a significant increase in IP3 accumulation and cellular hypertrophy. These responses were due to enhanced Gq-mediated signaling via Mas receptor [38]. Whether this response is a consequence of exacerbated Mas signaling or is a result of promiscuous signaling activation caused, for example, by heterodimerization, it is a topic that needs further clarification. Therefore, understanding the role of "physiological" versus "supraphysiological" levels of ACE2 and Mas, its downstream signaling pathways and their functional outcomes are crucial for clarifying the role of ACE2/Ang-(1-7)/Mas axis for cardiomyocyte function.

\section{Conclusions and Perspectives}

In cardiomyocytes, the actual knowledge of Ang-(1-7) protective effects was mainly focused on the modulation of Ang II signaling with emphasis on anti-hypertrophic actions. This effect was dependent on Mas, indicating that important 


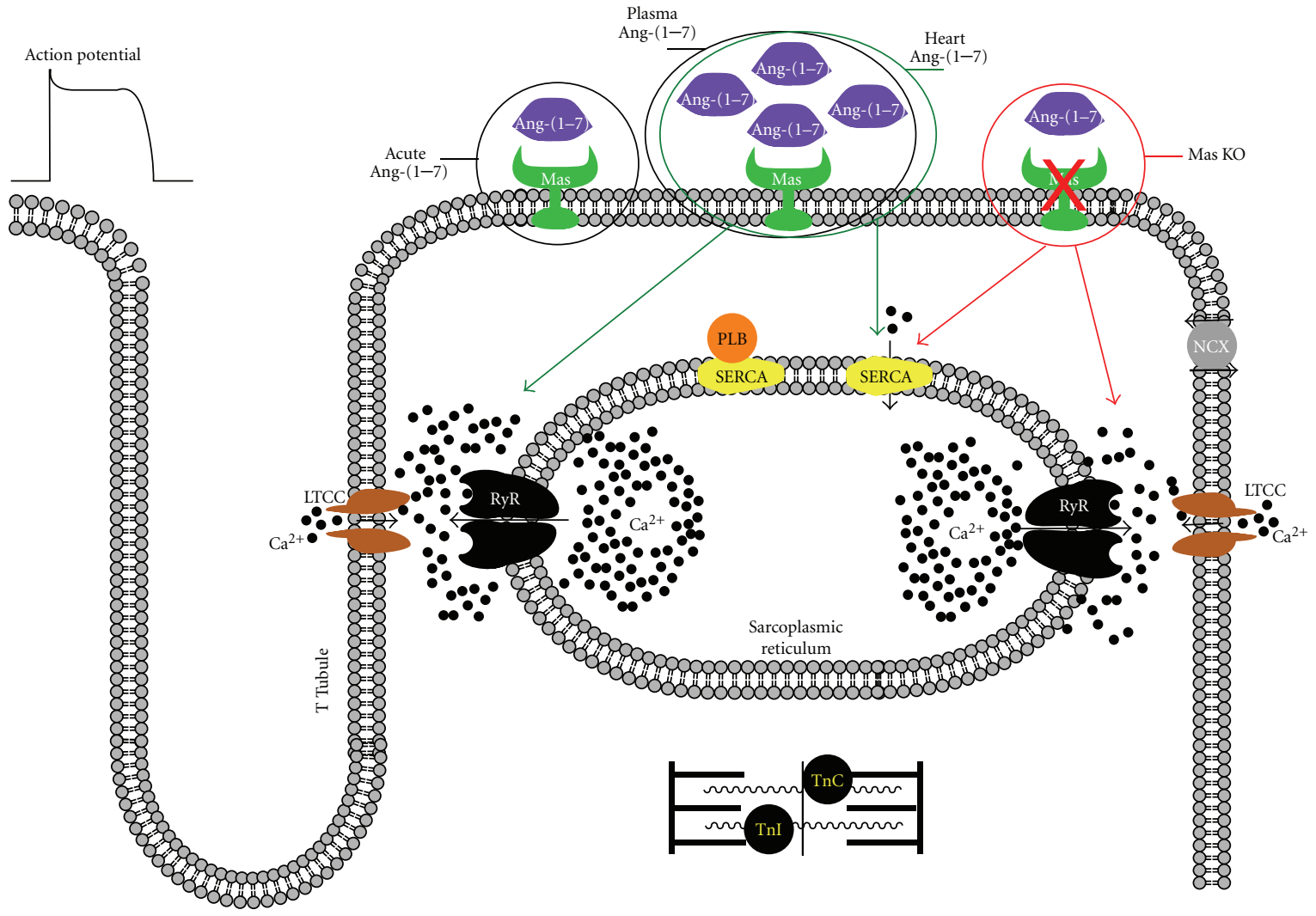

FIGURE 4: Summary of current knowledge regarding Ang-(1-7) modulation of $\mathrm{Ca}^{2+}$ handling in ventricular myocytes and the underlying mechanisms. Acute treatment of cardiomyocytes with Ang-(1-7) apparently has no direct effect on $\mathrm{Ca}^{2+}$ handling (black circle, left). Lack of effect on $\mathrm{Ca}^{2+}$ levels was also observed in cardiomyocytes from TG rats with increased circulating levels of Ang-(1-7) (black circle, middle). In contrast, Ang-(1-7) signaling disruption through Mas genetic ablation (Mas KO) leads to Ca ${ }^{2+}$ dysfunction (red circle, right). Cardiomyocytes from Mas KO mice present reduced SERCA expression levels and $\mathrm{Ca}^{2+}$ transients (red arrows). Cardiac specific overexpression of Ang-(1-7) enhances $\mathrm{Ca}^{2+}$ release and SERCA levels in ventricular myocytes (green circle and arrows). Data regarding Ang-(1-7) modulation of some other key proteins involved in $\mathrm{Ca}^{2+}$ handling in ventricular myocytes, such as PLN, NCX, TnI, and TnC, are still missing. Black filled circles = calcium ions; $\mathrm{LTCC}=\mathrm{L}$-type $\mathrm{Ca}^{2+}$ channels; Mas = Mas receptor; $\mathrm{NCX}=\mathrm{Na}^{+} / \mathrm{Ca}^{2+}$ exchanger; PLB $=$ phospholamban; $\mathrm{RyR}=$ ryanodine receptor; $\mathrm{TnC}=$ troponin $\mathrm{C} ; \mathrm{TnI}=$ troponin $\mathrm{I}$.

cardioprotective aspects of Ang-(1-7) signaling are mediated through Mas receptor, and involved NO and cGMP generation. It remains to be elucidated whether antioxidative and anti-inflammatory responses of Ang-(1-7) also depend on $\mathrm{NO} / \mathrm{cGMP}$ production. A direct acute effect of Ang-(1-7) on $\mathrm{Ca}^{2+}$ signaling in cardiomyocytes seems unlikely. However, there still are many ways by which Ang-(1-7) may regulate $\mathrm{Ca}^{2+}$ signaling in ventricular myocytes. Chronic increase in local Ang-(1-7) levels could be a mechanism by which Ang(1-7) enhances $\mathrm{Ca}^{2+}$ handling, as observed in cardiomyocytes from transgenic rats with cardiac specific overexpression of Ang-(1-7). To understand how Ang-(1-7) regulates $\mathrm{Ca}^{2+}$ handling in ventricular myocytes is of fundamental importance in light of Ang-(1-7) therapeutic potential in several disease conditions. The literature shows a tight control of ACE2 synthesis and activity in cardiomyocytes. How this enzyme expression is modulated, the signaling pathways involved in this regulation, and whether this occurs in vivo are questions that remain to be answered, considering the pivotal role of ACE2 as modulator of Ang II/Ang-(1-7) levels. The actions of Ang-(1-7) on cardiomyocytes are just beginning to unravel, dissecting the signaling pathways, and the conditions under which Ang-(1-7) signaling is turned on will be a major issue to be addressed in the future.

\section{References}

[1] A. V. Chobanian, "The hypertension paradox - More uncontrolled disease despite improved therapy," The New England Journal of Medicine, vol. 361, no. 9, pp. 848-887, 2009.

[2] T. Unger, "The role of the renin-angiotensin system in the development of cardiovascular disease," American Journal of Cardiology, vol. 89, no. 2, pp. 3A-10A, 2002.

[3] M. de Gasparo, K. J. Catt, T. Inagami, J. W. Wright, and T. Unger, "International union of pharmacology. XXIII. The angiotensin II receptors," Pharmacological Reviews, vol. 52, no. 3, pp. 415-472, 2000.

[4] M. Volpe, B. Musumeci, P. De Paolis, C. Savoia, and A. Morganti, "Angiotensin II AT2 receptor subtype: an uprising frontier in cardiovascular disease?" Journal of Hypertension, vol. 21, no. 8, pp. 1429-1443, 2003. 
[5] B. I. Levy, "How to explain the differences between renin angiotensin system modulators," American Journal of Hypertension, vol. 18, no. 9, part 2, pp. 134S-141S, 2005.

[6] U. M. Steckelings, R. E. Widdop, L. Paulis, and T. Unger, "The angiotensin AT2 receptor in left ventricular hypertrophy," Journal of Hypertension, vol. 28, no. 1, pp. S50-S55, 2010.

[7] R. A. S. Santos, K. B. Brosnihan, M. C. Chappell et al., "Converting enzyme activity and angiotensin metabolism in the dog brainstem," Hypertension, vol. 11, no. 2, part 2, pp. I153-I157, 1988.

[8] M. T. Schiavone, R. A. S. Santos, K. B. Brosnihan, M. C. Khosla, and C. M. Ferrario, "Release of vasopressin from the rat hypothalamo-neurohypophysial system by angiotensin(1-7) heptapeptide," Proceedings of the National Academy of Sciences of the United States of America, vol. 85, no. 11, pp. 4095-4098, 1988.

[9] M. J. Campagnole-Santos, D. I. Diz, R. A. S. Santos, M. C. Khosla, K. B. Brosnihan, and C. M. Ferrario, "Cardiovascular effects of angiotensin-(1-7) injected into the dorsal medulla of rats," American Journal of Physiology, vol. 257, no. 1, part 2, pp. H324-H329, 1989.

[10] I. F. Benter, D. I. Diz, and C. M. Ferrario, "Cardiovascular actions of angiotensin(1-7)," Peptides, vol. 14, no. 4, pp. 679684, 1993.

[11] I. F. Benter, D. I. Diz, and C. M. Ferrario, "Pressor and reflex sensitivity is altered in spontaneously hypertensive rats treated with angiotensin-(1-7)," Hypertension, vol. 26, no. 6, pp. 1138-1144, 1995.

[12] R. M. Carey and H. M. Siragy, "Newly recognized components of the renin-angiotensin system: potential roles in cardiovascular and renal regulation," Endocrine Reviews, vol. 24, no. 3, pp. 261-271, 2003.

[13] A. J. Ferreira, R. A. S. Santos, C. N. Bradford et al., "Therapeutic implications of the vasoprotective axis of the reninangiotensin system in cardiovascular diseases," Hypertension, vol. 55, no. 2, pp. 207-213, 2010.

[14] R. A. S. Santos, M. J. Campagnole-Santos, and S. P. Andrade, "Angiotensin-(1-7): an update," Regulatory Peptides, vol. 91, no. $1-3$, pp. 45-62, 2000.

[15] J. Varagic, A. J. Trask, J. A. Jessup, M. C. Chappell, and C. M. Ferrario, "New angiotensins," Journal of Molecular Medicine, vol. 86, no. 6, pp. 663-671, 2008.

[16] C. Vickers, P. Hales, V. Kaushik et al., "Hydrolysis of biological peptides by human angiotensin-converting enzyme-related carboxypeptidase," The Journal of Biological Chemistry, vol. 277, no. 17, pp. 14838-14843, 2002.

[17] L. S. Zisman, G. E. Meixell, M. R. Bristow, and C. C. Canver, "Angiotensin-(1-7) formation in the intact human heart: in vivo dependence on angiotensin II as substrate," Circulation, vol. 108, no. 14, pp. 1679-1681, 2003.

[18] D. J. Campbell, C. J. Zeitz, M. D. Esler, and J. D. Horowitz, "Evidence against a major role for angiotensin converting enzyme-related carboxypeptidase (ACE2) in angiotensin peptide metabolism in the human coronary circulation," Journal of Hypertension, vol. 22, no. 10, pp. 1971-1976, 2004.

[19] G. I. Rice, D. A. Thomas, P. J. Grant, A. J. Turner, and N. M. Hooper, "Evaluation of angiotensin-converting enzyme (ACE), its homologue ACE2 and neprilysin in angiotensin peptide metabolism," Biochemical Journal, vol. 383, no. 1, part 1, pp. 45-51, 2004.

[20] L. Stanziola, L. J. Greene, and R. A. S. Santos, "Effect of chronic angiotensin converting enzyme inhibition on angiotensin I and bradykinin metabolism in rats," American Journal of Hypertension, vol. 12, no. 10, part 1, pp. 1021-1029, 1999.
[21] P. E. Gallagher, C. M. Ferrario, and E. A. Tallant, "Regulation of ACE2 in cardiac myocytes and fibroblasts," American Journal of Physiology, vol. 295, no. 6, pp. H2373-H2379, 2008.

[22] M. Yamamuro, M. Yoshimura, M. Nakayama et al., "Aldosterone, but not angiotensin II, reduces angiotensin converting enzyme 2 gene expression levels in cultured neonatal rat cardiomyocytes," Circulation Journal, vol. 72, no. 8, pp. 13461350, 2008.

[23] J. Fielitz, A. Dendorfer, R. Pregla et al., "Neutral endopeptidase is activated in cardiomyocytes in human aortic valve stenosis and heart failure," Circulation, vol. 105, no. 3, pp. 286-289, 2002.

[24] E. Harada, M. Yoshimura, H. Yasue et al., "Aldosterone induces angiotensin-converting-enzyme gene expression in cultured neonatal rat cardiocytes," Circulation, vol. 104, no. 2, pp. 137139, 2001.

[25] Y. Zhang, L. J. Bloem, L. Yu et al., "Protein kinase C $\beta I I$ activation induces angiotensin converting enzyme expression in neonatal rat cardiomyocytes," Cardiovascular Research, vol. 57, no. 1, pp. 139-146, 2003.

[26] S. Hokimoto, H. Yasue, K. Fujimoto et al., "Expression of angiotensin-converting enzyme in remaining viable myocytes of human ventricles after myocardial infarction," Circulation, vol. 94, no. 7, pp. 1513-1518, 1996.

[27] C. A. M. van Kesteren, J. J. Saris, D. H. W. Dekkers et al., "Cultured neonatal rat cardiac myocytes and fibroblasts do not synthesize renin or angiotensinogen: evidence for stretch-induced cardiomyocyte hypertrophy independent of angiotensin II," Cardiovascular Research, vol. 43, no. 1, pp. 148-156, 1999.

[28] S. R. Tipnis, N. M. Hooper, R. Hyde, E. Karran, G. Christie, and A. J. Turner, "A human homolog of angiotensin-converting enzyme: cloning and functional expression as a captoprilinsensitive carboxypeptidase," The Journal of Biological Chemistry, vol. 275, no. 43, pp. 33238-33243, 2000.

[29] M. Donoghue, F. Hsieh, E. Baronas et al., "A novel angiotensin-converting enzyme-related carboxypeptidase (ACE2) converts angiotensin I to angiotensin 1-9," Circulation Research, vol. 87, no. 5, pp. E1-9, 2000.

[30] R. A. S. Santos, A. C. Simoes e Silva, C. Maric et al., "angiotensin-(1-7) is an endogenous ligand for the G protein-coupled receptor Mas," Proceedings of the National Academy of Sciences of the United States of America, vol. 100, no. 14, pp. 8258-8263, 2003.

[31] A. J. Ferreira and R. A. S. Santos, "Cardiovascular actions of angiotensin-(1-7)," Brazilian Journal of Medical and Biological Research, vol. 38, no. 4, pp. 499-507, 2005.

[32] S. M. Bindom and E. Lazartigues, "The sweeter side of ACE2: physiological evidence for a role in diabetes," Molecular and Cellular Endocrinology, vol. 302, no. 2, pp. 193-202, 2009.

[33] C. M. Ferrarlo, "ACE2: more of Ang-(1-7) or less Ang II?" Current Opinion in Nephrology and Hypertension, vol. 20, no. 1, pp. 1-6, 2011.

[34] A. J. Ferreira, V. Shenoy, Y. Yamazato et al., "Evidence for angiotensin-converting enzyme 2 as a therapeutic target for the prevention of pulmonary hypertension," American Journal of Respiratory and Critical Care Medicine, vol. 179, no. 11, pp. 1048-1054, 2009.

[35] D. Rakuan, M. Bürgelová, I. Vanková et al., "Knockout of angiotensin 1-7 receptor mas worsens the course of two-kidney, one-clip goldblatt hypertension: roles of nitric oxide deficiency and enhanced vascular responsiveness to angiotensin II," Kidney and Blood Pressure Research, vol. 33, no. 6, pp. 476-488, 2010. 
[36] M. F. Dias-Peixoto, R. A. S. Santos, E. R. M. Gomes et al., "Molecular mechanisms involved in the angiotensin-(17)/mas signaling pathway in cardiomyocytes," Hypertension, vol. 52, no. 3, pp. 542-548, 2008.

[37] E. R. M. Gomes, A. A. Lara, P. W. M. Almeida et al., "Angiotensin-(1-7) prevents cardiomyocyte pathological remodeling through a nitric oxide/guanosine 3',5'-cyclic monophosphate-dependent pathway," Hypertension, vol. 55, no. 1, pp. 153-160, 2010.

[38] T. Zhang, Z. Li, H. Dang et al., "Inhibition of Mas G-protein signaling improves coronary blood flow, reduces myocardial infarct size and provides long-term cardioprotection," American Journal of Physiology Heart and Circulatory Physiology, vol. 302, no. 1, pp. H299-311, 2012.

[39] E. A. Tallant, C. M. Ferrario, and P. E. Gallagher, "Angiotensin(1-7) inhibits growth of cardiac myocytes through activation of the mas receptor," American Journal of Physiology, vol. 289, no. 4, pp. H1560-H1566, 2005.

[40] R. A. S. Santos, C. H. Castro, E. Gava et al., "Impairment of in vitro and in vivo heart function in angiotensin-(1-7) receptor mas knockout mice," Hypertension, vol. 47, no. 5, pp. 996$1002,2006$.

[41] W. O. Sampaio, R. A. S. Dos Santos, R. Faria-Silva, L. T. da Mata Machado, E. L. Schiffrin, and R. M. Touyz, "Angiotensin(1-7) through receptor Mas mediates endothelial nitric oxide synthase activation via Akt-dependent pathways," Hypertension, vol. 49, no. 1, pp. 185-192, 2007.

[42] C. Vecchione, E. Patrucco, G. Marino et al., "Protection from angiotensin II-mediated vasculotoxic and hypertensive response in mice lacking PI3K $\gamma$," Journal of Experimental Medicine, vol. 201, no. 8, pp. 1217-1228, 2005.

[43] S. Wenzel, Y. Abdallah, S. Helmig, C. Schäfer, H. M. Piper, and K. -D. Schlüter, "Contribution of PI 3-kinase isoforms to angiotensin II- and $\alpha$-adrenoceptor-mediated signalling pathways in cardiomyocytes," Cardiovascular Research, vol. 71, no. 2, pp. 352-362, 2006.

[44] T. A. McKinsey and E. N. Olson, "Toward transcriptional therapies for the failing heart: chemical screens to modulate genes," The Journal of Clinical Investigation, vol. 115, no. 3, pp. 538-546, 2005.

[45] J. Heineke and J. D. Molkentin, "Regulation of cardiac hypertrophy by intracellular signalling pathways," Nature Reviews Molecular Cell Biology, vol. 7, no. 8, pp. 589-600, 2006.

[46] B. J. Wilkins and J. D. Molkentin, "Calcium-calcineurin signaling in the regulation of cardiac hypertrophy," Biochemical and Biophysical Research Communications, vol. 322, no. 4, pp. 1178-1191, 2004.

[47] C. L. Antos, T. A. McKinsey, N. Frey et al., "Activated glycogen synthase- $3 \beta$ suppresses cardiac hypertrophy in vivo," Proceedings of the National Academy of Sciences of the United States of America, vol. 99, no. 2, pp. 907-912, 2002.

[48] M. Flores-Muñoz, N. J. Smith, C. Haggerty, G. Milligan, and S. A. Nicklin, "Angiotensin1-9 antagonises pro-hypertrophic signalling in cardiomyocytes via the angiotensin type 2 receptor," Journal of Physiology, vol. 589, part 4, pp. 939-951, 2011.

[49] Y. Qi, V. Shenoy, F. Wong et al., "Lentivirus-mediated overexpression of angiotensin-(1-7) attenuated ischaemia-induced cardiac pathophysiology," Experimental Physiology, vol. 96, no. 9, pp. 863-874, 2011.

[50] D. M. Bers, "Cardiac excitation-contraction coupling," Nature, vol. 415, no. 6868, pp. 198-205, 2002.

[51] A. J. Ferreira, C. H. Castro, S. Guatimosim et al., "Attenuation of isoproterenol-induced cardiac fibrosis in transgenic rats harboring an angiotensin-(1-7)-producing fusion protein in the heart," Therapeutic Advances in Cardiovascular Disease, vol. 4, no. 2, pp. 83-96, 2010.

[52] J. Zhong, R. Basu, D. Guo et al., "Angiotensin-converting enzyme 2 suppresses pathological hypertrophy, myocardial fibrosis, and cardiac dysfunction," Circulation, vol. 122, no. 7, pp. 717-728, 2010.

[53] R. Masson, S. A. Nicklin, M. A. Craig et al., "Onset of experimental severe cardiac fibrosis is mediated by overexpression of Angiotensin-converting enzyme 2," Hypertension, vol. 53, no. 4, pp. 694-700, 2009. 


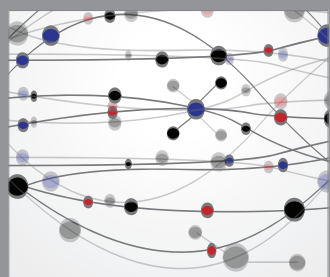

The Scientific World Journal
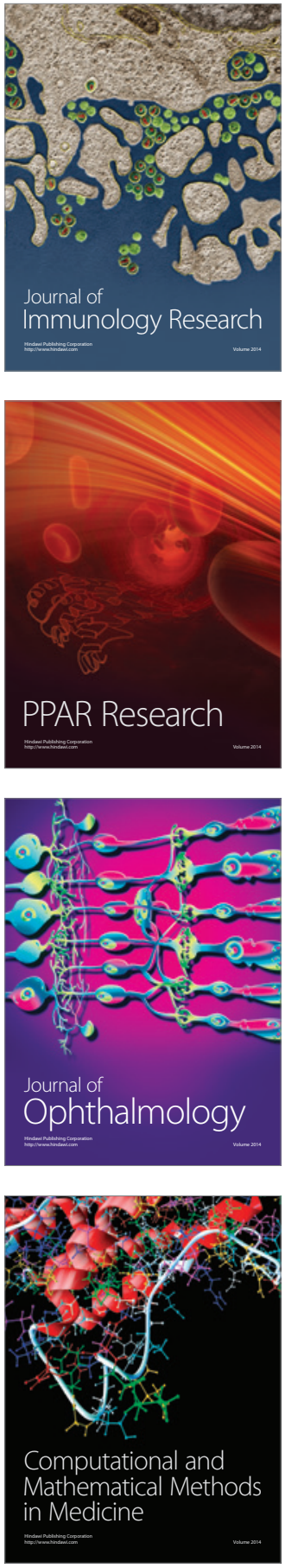

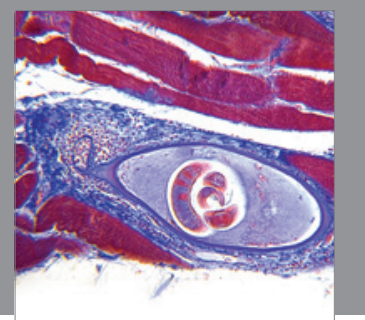

Gastroenterology

Research and Practice
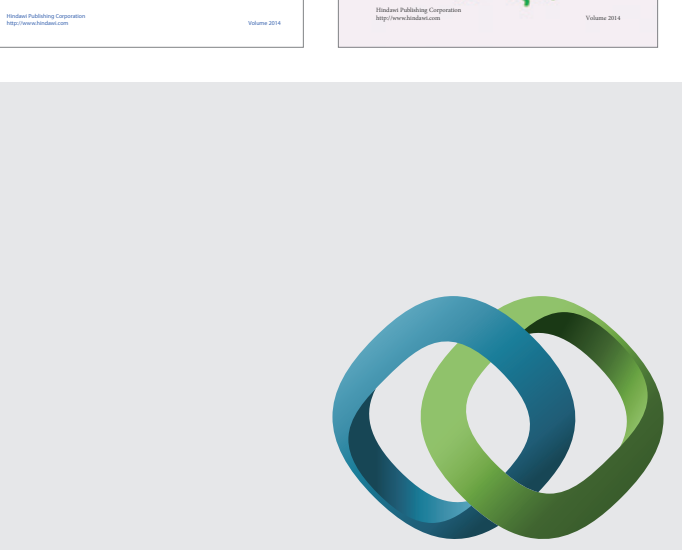

\section{Hindawi}

Submit your manuscripts at

http://www.hindawi.com
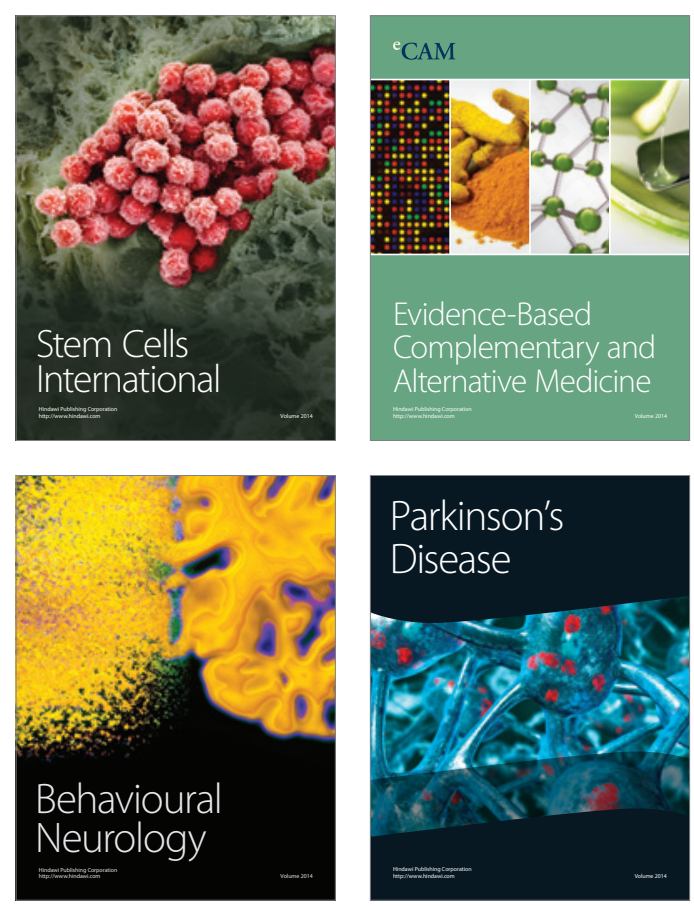

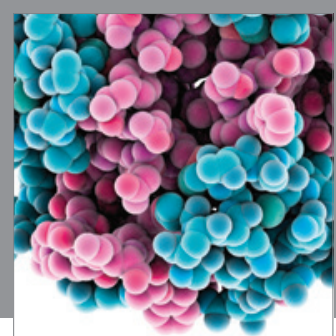

Journal of
Diabetes Research

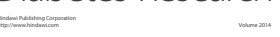

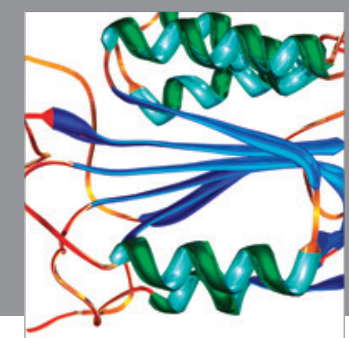

Disease Markers
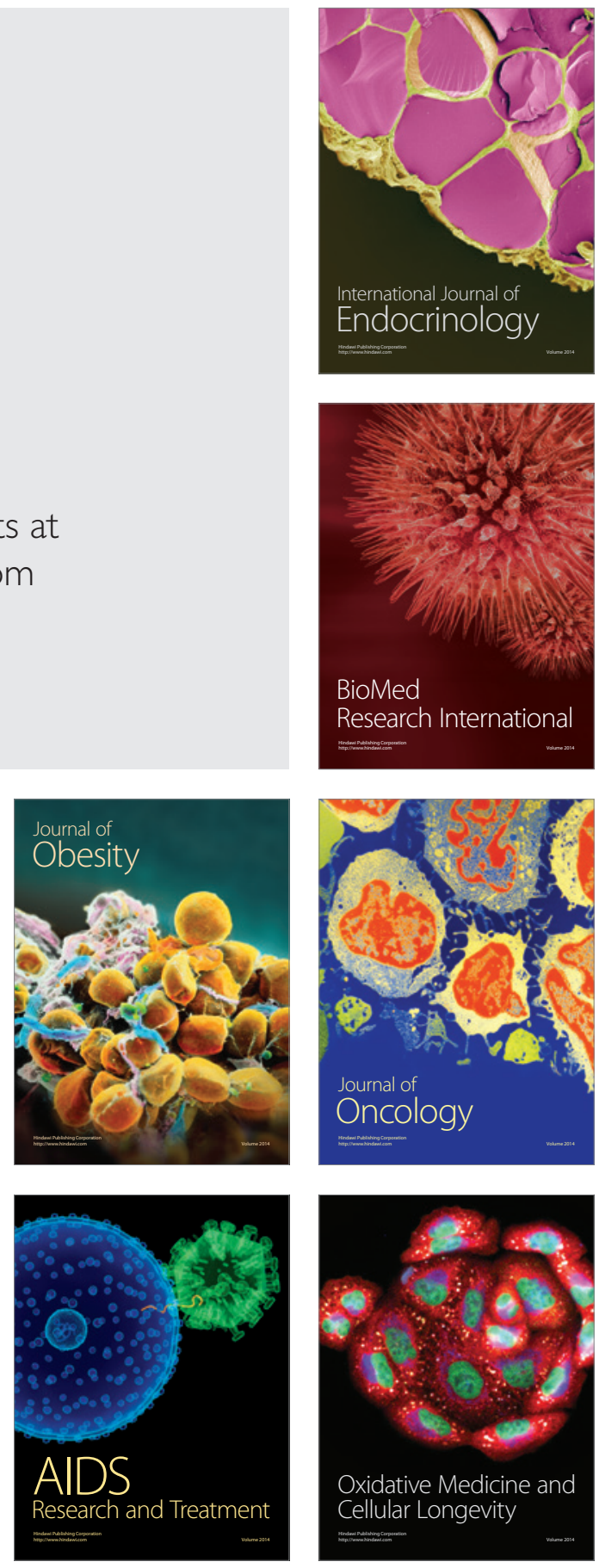